\title{
Research in Energy Harvesting Wireless Sensor Networks and the Challenges Ahead
}

\author{
Winston K.G. Seah, Y.K. Tan and Alvin T.S. Chan
}

\begin{abstract}
Wireless sensor networks (WSNs) are set to form a significant part of the new pervasive Internet, often referred to as the Internet of Things. WSNs have traditionally been powered by limited energy sources, viz. batteries, limiting their operational lifetime. To ensure the sustainability of WSNs, researchers have turned to alternative energy sources for power. Harvesting ambient energy from the environment to power WSNs is a promising approach but it is currently unable to provide a sustained energy supply to support continuous operation. Sensor nodes therefore need to exploit the sporadic availability of energy to quickly sense and transmit the data. We first review the recent developments in energy harvesting technology and research on networking protocol design for Wireless Sensor Networks Powered by Ambient Energy Harvesting. Then, we discuss some of the challenges faced by researchers in designing networking protocols and summarize the open research problems.
\end{abstract}

Index Terms—Energy harvesting/scavenging, Protocol design, Wireless sensor network.

Winston K.G. Seah is with the School of Engineering and Computer Science, Victoria University of Wellington, P.O. Box 600, Wellington 6140, New Zealand. (e-mail: Winston.Seah@ecs.vuw.ac.nz)

Yen Kheng Tan is with the Energy Research Institute@ Nanyang Technological University (ERI@N), Research Techno Plaza, X-Frontier, Level 5, 50 Nanyang Drive, Singapore 637553. (email: tanyenkheng@ieee.org)

Alvin T.S. Chan is with the Department of Computing, The Hong Kong Polytechnic University, Hung Hom, Hong Kong. (email: cstschan@comp.polyu.edu.hk) 


\section{INTRODUCTION}

Wireless sensor networks (WSNs) are set to form a significant portion of the smart pervasive Internet of Things. Like the traditional Internet and many other technologies, defence applications have motivated the research in WSNs [1]. The often-cited deployment scenario for WSNs is that of a large number of small wireless sensor nodes randomly scattered over the area of interest to collect information on entities of interest. Research has predominantly assumed that sensor nodes are powered by a portable and limited energy source, viz., batteries. Once a sensor node's power supply is exhausted, it can no longer fulfill its role unless the source of energy is replenished. Therefore, it is generally accepted that the usefulness of a wireless sensor expires when its battery runs out. Much of the research on wireless sensor networks has therefore focused on efficient methods to minimize energy usage in order to extend the lifetime of the nodes that form the network.

Rapid technological progress has made available low-cost sensors and communication networks which led to the development of various other potential WSN applications [1]. A salient feature of these new applications is the need for sensors to operate for much longer durations (like years or even decades) after they are deployed, for example, in in-situ environmental/habitat monitoring and structural health monitoring of critical infrastructures and buildings, where batteries are hard (or even impossible) to replace after the sensors are deployed. Recently, alternative energy sources for WSNs are actively investigated. Of particular interest is the harvesting of ambient energy from the environment and converting it into electricity to power the sensor nodes. While renewable energy technology is not new (e.g., solar and wind) the systems in use are far too large for WSNs. Those small enough for use in wireless sensors are unlikely to provide a sustained supply to support continuous operation. They are likely to be able to provide only enough energy to power sensors sporadically and sensor nodes therefore need to exploit the sporadic availability of energy to quickly sense and transmit the data.

In this paper, we first discuss the concept of a wireless sensor network in which sensor nodes rely solely on harvested energy for power, referred to as Wireless Sensor Networks Powered by Ambient Energy Harvesting, or WSN-HEAP for short [2], and not hybrid systems that use energy harvesting to supplement batteries [3][4]. Since then, there have been active research efforts by both the academia as well as the industry in this area; for earlier work reported in the literature, the reader can refer to [2] and the references therein. We then review the developments in energy harvesting systems and protocol design. Before concluding, we discuss the open research 
problems and challenges ahead that need to be addressed.

\section{OVERVIEW OF WSN-HEAP CONCEPT}

In WSN-HEAP, each sensor node uses one or more energy harvesting devices to harvest ambient energy, such as light, vibration, and heat, from the environment and stores the harvested energy in a storage device. The differences in the system architecture between a battery-powered wireless sensor node and WSN-HEAP node are shown in Figure 1. Harvesting energy from the environment is not new and has been in use for decades. The most common forms of ambient energy include water (hydro-electric power generation), light (solar panels), wind (wind turbines) and thermal (particularly in areas with volcanic activities). Harvesting energy for low-power (and possibly embedded) devices like wireless sensors presents a new challenge as the energy harvesting device has to be comparable in size (i.e. small enough) with the sensors. Furthermore, the placement of the sensors may not be in locations that will achieve optimal energy harvesting performance. There are complex trade-offs to be considered when designing energy harvesting systems for WSNs arising from the interaction of various factors like the characteristics of the energy sources, energy storage device(s) used, power management functionality of the nodes and protocols, and the applications' requirements. Currently, the main sources of ambient energy considered suitable for use with WSNs are solar, mechanical (vibration or strain), thermal and electromagnetic energy [5].

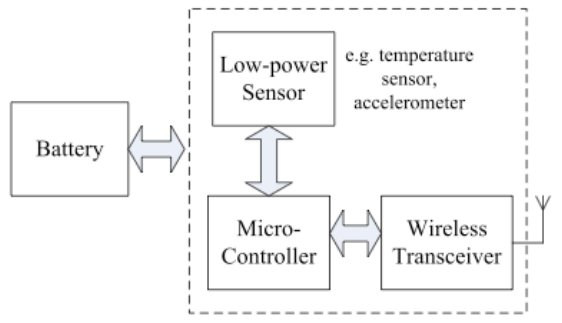

(a) Battery-operated wireless sensor

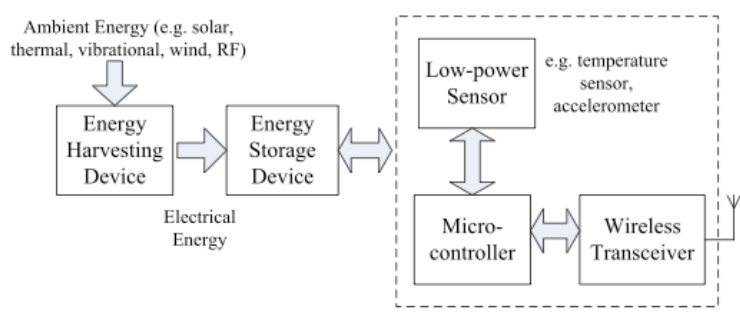

(b) WSN-HEAP node

Figure 1. Battery-operated Wireless Sensor vs WSN-HEAP node.

Besides the energy harvesting component, another critical component of a WSN-HEAP node is the energy storage device. Baring wear-and-tear and other forms of physical damage, the goal is to substantially minimize, or ideally, totally eliminate the need to physically replace the energy storage device or manually replenish the energy. The primary candidates for energy storage in WSN-HEAP are rechargeable battery and super-capacitor. The key advantage that the super-capacitor has over the rechargeable battery is its virtually unlimited recharge cycles - in the 
order of a million cycles as compared to less than 1000 cycles for rechargeable batteries. This makes supercapacitors the more viable energy storage option for WSN-HEAP. Issues in storage devices and low-power electronics design that are suitable for use in WSN-HEAP are discussed in [6].

The energy characteristics of a WSN-HEAP node are distinctly different from that of a battery-powered wireless sensor node, as illustrated in Figure 2. In a battery-powered node, the total energy reduces over time and the sensor remains operational until the energy level drops to an unusable level. At this stage, either the battery needs to be replaced or the node is deemed to be unusable/dead. On the other hand, the energy in a WSN-HEAP node is replenished with energy harvested from the environment. The energy needs to be accumulated over time until a certain level (e.g. $E_{\min }$ in Figure 2) before it can be used. Due to technology limitations and the unpredictable nature of the environment, the rate of harvesting and charging is unlikely to be able to support continuous sustained node operation. A WSN-HEAP node is normally awake and operating for a short duration before it needs to shut down to recharge. Similar cyclic trends (see Figure 3) have also been observed in experiments carried out with solar energy harvesting and reported in [7]. At the start of their experiments, power was drawn from Battery 1 until its voltage falls below 4V at sample 900; although Battery 2 was not used, it suffered from self-discharge and its voltage level dropped slightly over time. The system then switched Battery 1 to energy harvesting mode and started drawing power from Battery 2. Therefore, the voltage level of Battery 2 began falling at a faster rate until it dropped below $4 \mathrm{~V}$ at 2600. Meanwhile, solar energy harvesting had recharged Battery 1 to its capacity but while it was not used, it suffered from self-discharge, like Battery 2 previously. At sample 2600, the two batteries were switched over again. The voltage level of Battery 1 dropped faster as power was drawn from it, while Battery 2 was recharged. Hence, the study has validated the cyclic behaviour of the voltage levels shown in the conceptual model presented in Figure 2. A summary of key aspects and differences between battery-powered WSN and WSN-HEAP is provided in Table 1.

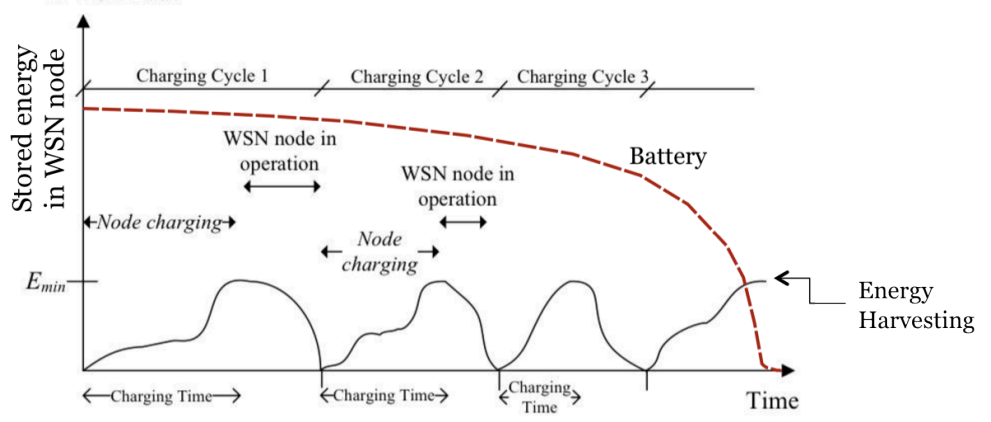

Figure 2. Energy characteristics of WSN-HEAP node. 


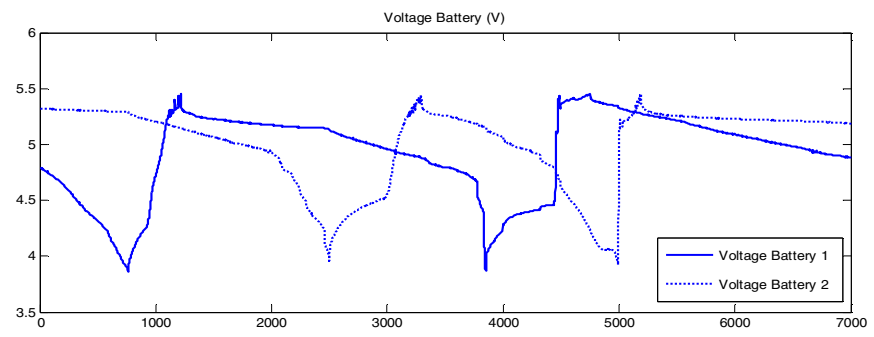

Figure 3. Energy Characteristics of Two-battery Solar Powered Sensor System [7]

\begin{tabular}{|c|c|c|c|}
\hline & Battery-operated WSNs & $\begin{array}{c}\text { Battery-operated WSNs supplemented } \\
\text { with Energy Harvesting }\end{array}$ & WSN-HEAP \\
\hline Goal & $\begin{array}{l}\text { Latency and throughput is } \\
\text { usually traded off for longer } \\
\text { network lifetime }\end{array}$ & $\begin{array}{l}\text { Longer lifetime is achieved since } \\
\text { battery power is supplemented by } \\
\text { harvested energy }\end{array}$ & $\begin{array}{l}\text { Maximize throughput and minimize } \\
\text { delay since energy is renewable } \backslash \& \\
\text { the traditional concept of network } \\
\text { lifetime does not apply }\end{array}$ \\
\hline $\begin{array}{l}\text { Protocol } \\
\text { Design }\end{array}$ & $\begin{array}{l}\text { Sleep-and-wakeup schedules } \\
\text { can be determined precisely }\end{array}$ & $\begin{array}{l}\text { Sleep-and-wakeup schedules can be } \\
\text { determined if predictions about future } \\
\text { energy availability are correct }\end{array}$ & $\begin{array}{l}\text { Sleep-and-wakeup schedules cannot } \\
\text { be predicted; difficult to know } \\
\text { exactly which is the awake next-hop } \\
\text { neighbor to forward data to }\end{array}$ \\
\hline $\begin{array}{l}\text { Energy } \\
\text { Model }\end{array}$ & $\begin{array}{l}\text { Energy model is well } \\
\text { understood }\end{array}$ & $\begin{array}{l}\text { Energy model can predicted with high } \\
\text { accuracy }\end{array}$ & $\begin{array}{l}\text { Energy harvesting rate varies across } \\
\text { time, space as well as the type of } \\
\text { energy harvesters used; energy } \\
\text { model is hard to predict }\end{array}$ \\
\hline
\end{tabular}

Table 1. Summary of Key Aspects and Differences between WSN and WSN-HEAP.

\section{ENERGY HARVESTING TECHNOLOGY}

To overcome the major hindrance of the "deploy and forget" nature of WSNs due to the limitation of available energy for the network constrained by the high power consumption of the sensor nodes and the energy capacity and unpredictable lifetime performance of the battery, EH technology has emerged as a promising solution to sustain the operation of WSN [8] [9].

\section{A. Overview of Renewable Energy Harvesting}

Energy harvesting (EH) is a technique that captures, harvests or scavenges a variety of unused ambient energy sources such as solar, thermal, vibration and wind, and converts the harvested energy into electrical energy to recharge the batteries. The harvested energy in WSNs is generally very small (of the order of $m J$ ) as compared to those large-scale EH applications using renewable energy sources such as solar farms and wind farms of the order of several hundreds MJ. Unlike the large-scale power stations that are fixed at a given location, the small-scale energy 
sources are portable and readily available for usage. Various energy harvesting sources excluding the biological type, which can be converted into electrical energy, are shown in Figure 4.

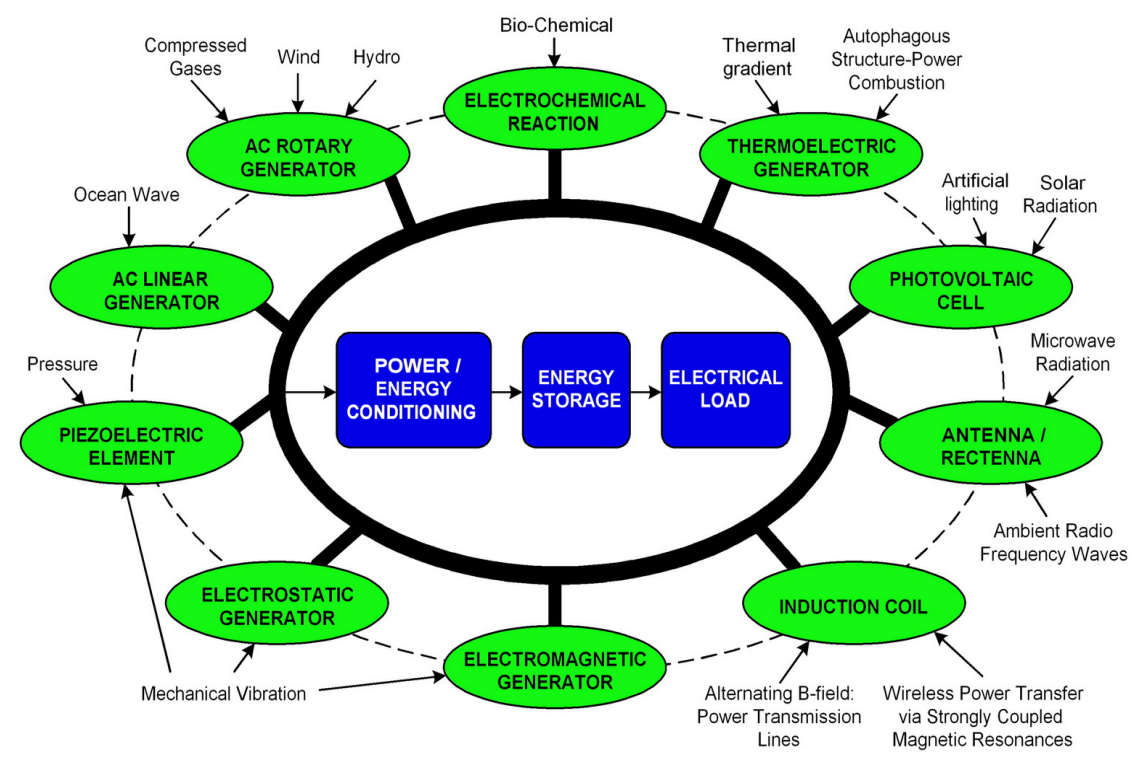

Figure 4. Energy harvesting sources and their energy harvesters, adapted from [10].

In our environment, there are full of wasted and unused ambient energy generated from these energy sources seen in Figure 4. These renewable energy sources are ample and readily available in the environment and so it is not necessary to deliberately expend efforts to create these energy sources like the example of burning the nonrenewable fossil fuels to create steam that in turn drives the steam turbines to create electrical energy. Unlike exhaustible fossil fuels, the majority of the environmental energy sources are renewable and sustainable for almost infinitely long periods. Numerous studies and experiments have been conducted to investigate the levels of energy that could be harvested from the ambient environment. A compilation list of various energy harvesting sources and their power/energy densities are listed in Table 2.

Table 2 shows the performance of each EH source in terms of the power density factor. It can be clearly observed that there is no unique solution suitable for all environments and applications. According to Table 2, it can be observed that solar energy source yields the highest power density. However, this may not be always the case. Under illuminated indoor condition, the ambient light energy harvested by the solar panel drops tremendously. The other energy harvesting sources could provide higher power density depending on the renewable energy sources available at the specific application areas like, outdoor bright sunny day with rich amount of solar energy, along coastal area 
with a lot of wind energy, bridge structure with vehicles travelling that has strong vibrations, etc. In addition, there could also be the possibility of two or more energy sources available for harvesting at the same time.

\begin{tabular}{|c|c|c|}
\hline Energy Source & Performance & Notes \\
\hline \multirow[t]{2}{*}{ Ambient light } & $\begin{array}{l}100 \mathrm{~mW} / \mathrm{cm}^{2} \text { (di- } \\
\text { rect sunlight) }\end{array}$ & \multirow[t]{2}{*}{$\begin{array}{l}\text { Common polycrystalline solar cells are } 16 \%-17 \\
\% \text { efficient, while standard mono-crystalline cells } \\
\text { approach } 20 \%\end{array}$} \\
\hline & $\begin{array}{l}100 \mu \mathrm{W} / \mathrm{cm}^{2} \text { (illu- } \\
\text { minated office) }\end{array}$ & \\
\hline Thermal & $\begin{array}{l}{ }^{\text {a) }} 60 \mu \mathrm{W} / \mathrm{cm}^{2} \text { at } 5 \mathrm{~K} \\
\text { gradient } \\
{ }^{b)} 135 \mu \mathrm{W} / \mathrm{cm}^{2} \text { at } 10 \\
\mathrm{~K} \text { gradient }\end{array}$ & $\begin{array}{l}\text { Typical efficiency of thermoelectric generators are } \\
\leq 1 \% \text { for } \Delta \mathrm{T}<313 \mathrm{~K} \\
{ }^{a)} \text { Seiko Thermic wristwatch at } 5 \mathrm{~K} \text { body heat, } \\
{ }^{b)} \text { Quoted for a ThermoLife }{ }^{\Theta} \text { generator at } \Delta \mathrm{T}= \\
10 \mathrm{~K}\end{array}$ \\
\hline Blood Pressure & $0.93 \mathrm{~W}$ at $100 \mathrm{mmHg}$ & $\begin{array}{l}\text { When coupled with piezoelectric generators, the } \\
\text { power that can be generated is order of } \mu \mathrm{W} \text { when } \\
\text { loaded continuously and } \mathrm{mW} \text { when loaded inter- } \\
\text { mittently }\end{array}$ \\
\hline Vibration & $\begin{array}{l}4 \mu \mathrm{W} / \mathrm{cm}^{3} \quad(\mathrm{hu}- \\
\operatorname{man} \text { motion-Hz) } \\
800 \mu \mathrm{W} / \mathrm{cm}^{3} \\
(\text { machines-kHz) }\end{array}$ & $\begin{array}{l}\text { Predictions for } 1 \mathrm{~cm}^{3} \text { generators. Highly depen- } \\
\text { dent on excitation (power tends to be proportional } \\
\text { to } \omega \text {, the driving frequency and } \mathrm{y}_{o} \text {, the input dis- } \\
\text { placement }\end{array}$ \\
\hline $\begin{array}{l}\text { Hand Linear } \\
\text { Generator }\end{array}$ & $2 \mathrm{~mW} / \mathrm{cm}^{3}$ & Shake-driven flashlight of $3 \mathrm{~Hz}$ \\
\hline Push button & $50 \mu \mathrm{J} / \mathrm{N}$ & Quoted at $3 \mathrm{~V}$ DC for the MIT Media Lab Device \\
\hline Heel strike & $118 \mathrm{~J} / \mathrm{cm}^{3}$ & Per walking step on piezoelectric insole \\
\hline Ambient wind & $1 \mathrm{~mW} / \mathrm{cm}^{2}$ & $\begin{array}{l}\text { Typical average wind speed of } 3 \mathrm{~m} / \mathrm{s} \text { in the ambi- } \\
\text { ent }\end{array}$ \\
\hline $\begin{array}{l}\text { Ambient radio } \\
\text { frequency }\end{array}$ & $<1 \mu \mathrm{W} / \mathrm{cm}^{2}$ & Unless near a RF transmitter \\
\hline $\begin{array}{l}\text { Wireless energy } \\
\text { transfer }\end{array}$ & $14 \mathrm{~mW} / \mathrm{cm}^{2}$ & Separation distance of 2 metres \\
\hline
\end{tabular}

Table 2. Energy harvesting opportunities and demonstrated capabilities adapted from [11].

As such, EH technology can provide numerous benefits to the end user and some of the major benefits about EH suitable for WSN are stated and elaborated in the following list. Energy harvesting solutions can:

1. Reduce the dependency on battery power - with the advancement of microelectronics technology, the power consumption of the sensor nodes are getting lesser and lesser, hence harvested ambient/environmental energy may be sufficient to eliminate the need for batteries completely.

2. Reduce installation cost - self-powered wireless sensor nodes do not require power cables wiring and conduits, hence they are very easy to install and also reduce the heavy installation cost. 
3. Reduce maintenance cost - energy harvesting allows for the sensor nodes to function unattended once deployed and eliminates service visits to replace batteries.

4. Provide sensing and actuation capabilities - especially in hard-to-access hazardous environments on a continuous basis.

5. Provide long-term solutions - a reliable self-powered sensor node will remain functional virtually as long as the ambient energy is available. Self-powered sensor nodes are perfectly suited for long-term applications looking at decades of monitoring.

6. Reduce environmental impact - energy harvesting can eliminate the need for millions on batteries and energy costs of battery replacements.

Clearly, it can be deduced from the list of benefits that EH technology is a viable solution to power WSNs and mobile devices for extended operation with the supplement of the energy storage devices, if not completely eliminating the storage devices such as batteries.

The latest trend in energy harvesting involves bioenergy, which is renewable energy derived from biological sources. The energy in oxygen and glucose molecules in blood has become the target of bioengineers looking for an energy source to power implantable devices without having to use batteries [12]. Other sources of bioenergy include human and animal wastes, tree [13], etc.

\section{B. Modelling of Energy Harvesting Sources}

In order to design networking protocols for realistic WSN-HEAP applications, we need to characterize the charging time of energy harvesters and radio transmission behaviour of the EH system unit as shown in Figure 5. Most manufacturer datasheets of the energy harvesting devices only describe the average harvesting rates and not the charging characteristics. As the source of harvested energy comes from the environment, it is difficult to predict the time when the energy is available. Furthermore, the instantaneous energy level obtainable from the energy harvester is variable and also inadequate to operate a sensor node. To mitigate the unpredictability of the energy source, the harvested energy is stored in a buffer (e.g. super-capacitor) until there is sufficient to power the sensor node for the desired operations like sensing, receiving and/or transmitting packets. Important energy charging characteristics include charging time and number of packets that can be transmitted per charge cycle (cf: Figure 2). 


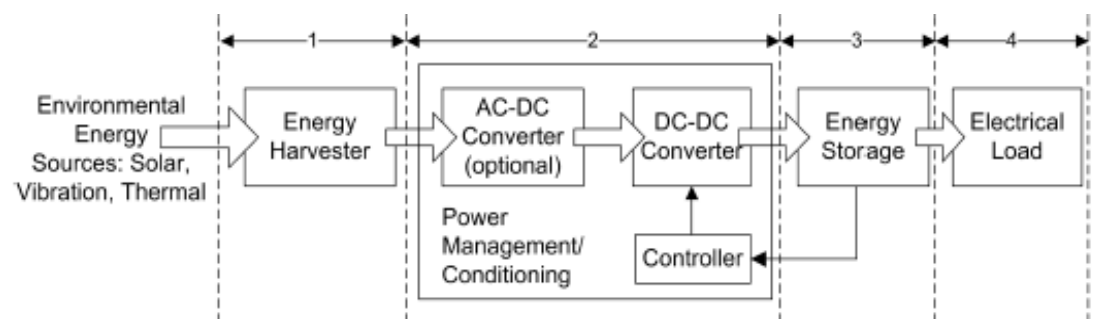

Figure 5. General block diagram representation of energy harvesting system unit.

A study to empirically characterize the charging times of commercially available solar energy harvesting devices for WSN-HEAP is reported in [21]. To measure the charging time, energy is accumulated by the harvester and stored in the storage device. When the energy level has reached a sufficient level ( $E_{\min }$ in Figure 2$)$, the microcontroller and transceiver on the sensor node are switched on. The transmitter then continuously broadcasts data packets until the energy is depleted, after which the microcontroller and transceiver are turned off. The energy storage device will start to accumulate energy again and the process is repeated in the next cycle.

Figure 6 shows the probability density functions (pdf) of the charging times for 1000 charge cycles for three deployment scenarios with the solar energy harvester placed directly under, $1 \mathrm{~m}$ and $2 \mathrm{~m}$ under a fluorescent lamp. The results show that there is greater variation (higher standard deviation) in the charging time required for each charge cycle when the sensor node is further away from the light source. Similar variations have also been observed with a thermal energy harvester mounted on a CPU heat sink.

The high variability in the energy harvesting process, in both time and space, has also been observed by another study [22] using a time-slotted solar energy harvesting node with different system and environmental parameters. Six different statistical models, viz., uniform distribution, geometric distribution, transformed geometric distribution, Poisson distribution, transformed Poisson distribution and a Markovian model, were used to fit the empirical datasets. The study concluded that no single statistical model can fit all the datasets, and empirical data is needed to validate the theoretical analysis of time-slotted MAC protocols for WSN-HEAP. 


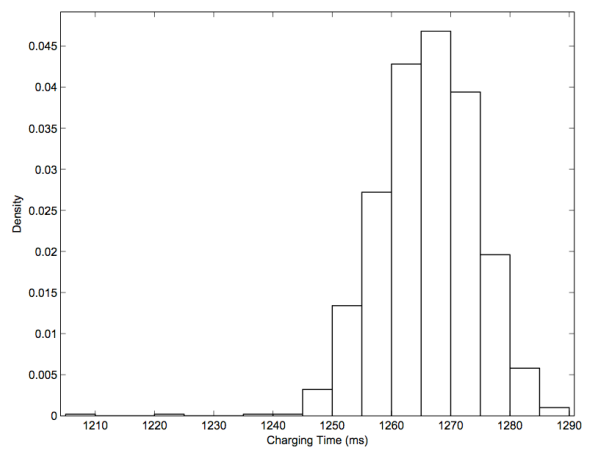

(a) Directly under lamp.

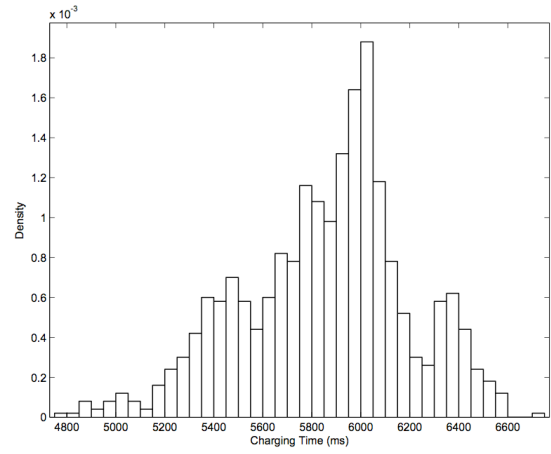

(b) $1 \mathrm{~m}$ under lamp.

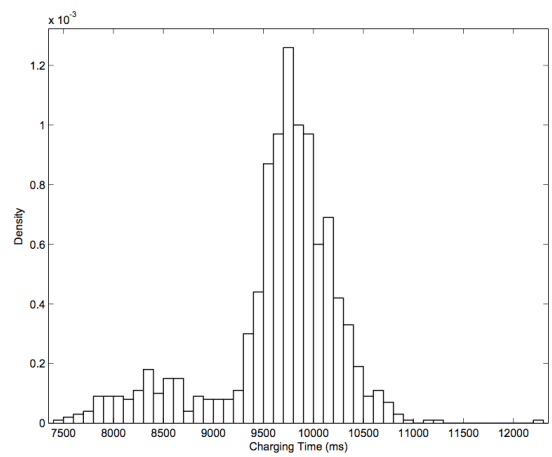

(c) $2 \mathrm{~m}$ under lamp

Figure 6. Solar Energy Harvesting Technology - Charging Times probability density functions [22].

Analytical models supported with empirical data are derived in [23]. The study involved harvesting solar as well as piezoelectric (PZ) energy. A solar harvesting module was deployed next to a glass window in a typical office building where it harvested energy from sunlight and indoor fluorescent light. Readings were recorded every thirty seconds over twenty-two days, giving about 65,000 samples. Typical measurement results of solar energy harvesting over a 24-hour period across five days are shown in Figure 7(a). The PZ film was clamped vertically and fixed to a plastic "wind catcher" such that the wind will cause the PZ film to oscillate back and forth. The oscillation depends on the strength and duration of the wind, resulting in an erratic supply of harvested energy, as shown in Figure 7(b). 


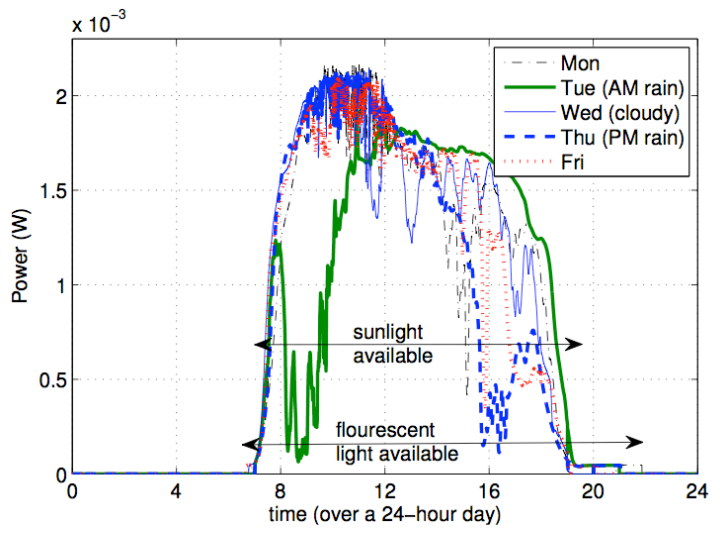

(a) Harvested solar energy over 5 days.

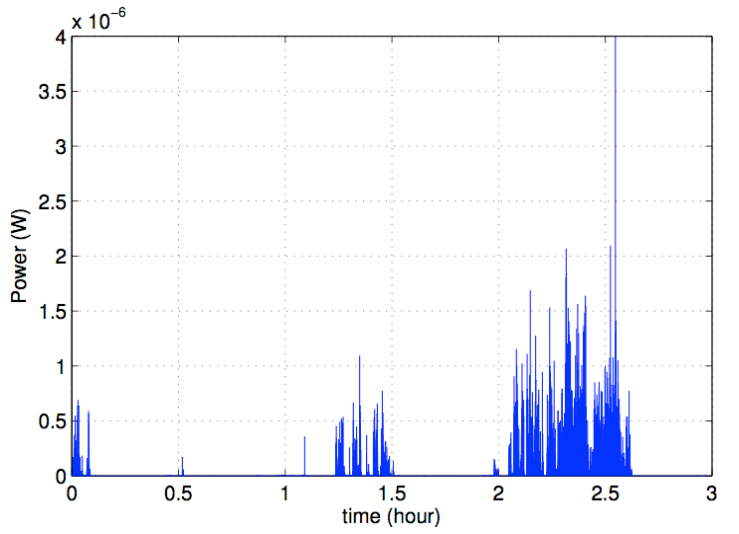

(b) Harvested PZ energy over 2 hours.

Figure 7. Harvested solar and PZ energy for analytical models [23].

The harvested energy level, denoted by $E_{k}$, where $k=1,2, \ldots$, is a non-stationary Markovian stochastic process over discrete time $k$. A scenario parameter, denoted by $S_{k}$ and modelled by a Markovian process, is introduced to characterize the different classes of energy harvesting environments so that, in each class, $E_{k}$ is a stationary process. The study suggested that a good choice of $S_{k}$ depends on the energy harvesting technology and other system parameters. It also proposed that a pragmatic choice of value for $S_{k}$ to predict the harvested energy is the past average harvested energy. Using Bayesian information criterion, the study concluded that PZ energy can be better modelled by the generalized Markovian model, while solar energy is usually better modelled by a stationary Markovian model.

\section{Design Principle of Wireless Sensor Network with Energy Harvesting Technology}

In an energy harvesting (EH) system, there are generally four main components, namely, energy collection and conversion mechanism (energy harvester), electrical power management/conditioning circuit, energy storage device and electrical load (wireless sensor node) as shown in Figure 5. Power output per unit mass or volume i.e. power/energy density is a key performance unit for the energy collection and conversion mechanisms. The harvested power must be converted into electricity and conditioned to an appropriate form for either charging the system capacitors/batteries or powering the connected load directly. Proper load impedance matching between the EH source and the electrical load is necessary to maximize the usage of the harvested energy. Appropriate electronic circuitry for power conditioning and load impedance matching may be available commercially or may require custom design and fabrication. 
Referring to Figure 5, it can be seen that the function of the energy harvester is to convert energy harnessed from environmental energy sources into electrical energy. Typical examples of the energy harvesters, as shown in Figure 4, include the Lead Zirconate Titanate (PZT) ceramic material that converts mechanical (strain or stress) energy into electrical energy due to the piezoelectric effect, the photovoltaic cell that converts solar energy into electrical energy, the thermoelectric generator that outputs electrical voltage when there is a thermal gradient across it and the wind turbine that converts kinetic energy from wind flow into electrical energy. The harvested electrical energy from the energy harvester needs to be conditioned by some form of power conditioning circuit before supplying it to the load. The main objective of the power electronics technology in the power conditioning circuit as seen in Figure 5 is to process and control the flow of electrical energy from the source to the load in such a way that energy is used efficiently. This matching process is a crucial step to ensure that maximum power is transferred from the source to the electrical load. Another function of the power conditioning circuit involves the conversion and regulation of electrical voltage at higher levels into suitable levels for the loads.

To ensure continuity in the load operation even when the external power source is weak or temporarily unavailable, the excess energy already being harvested earlier has to be stored either in the rechargeable battery or super-capacitor as shown in Figure 5. Depending on the environmental condition of the ambient energy source, the characteristic of the energy harvester and the power requirement of the load i.e. wireless sensor node and control circuitry, each individual energy harvesting system is designed and optimized accordingly in order to sustain the operation of the wireless sensor node.

\section{Networking Protocol Design}

This section reviews the recent research developments in networking protocol design since the introduction of the WSN-HEAP concept [2].

\section{A. Power and Topology Management}

The use of energy harvesting to power WSNs has been acknowledged as the solution for long-term applications where the option of replacing batteries to replenish the energy is impractical. The perennial problem of limited energy in battery-powered WSNs is more pronounced in WSN-HEAP where managing the energy resource is more difficult. Timing schedules are hard to control when there is no energy left and the time it takes to replenish the energy cannot be accurately predicted due to environmental conditions. Power management in battery-powered 
WSN typically addresses the problem of allocating power to the different components, and energy conservation is usually of utmost importance in the design of protocols. In WSN-HEAP, energy conservation may not necessarily be beneficial since the storage devices, viz. capacitors, are limited in capacity and leakage-prone. Therefore, the common approach adopted in WSNs of not transmitting/operating to conserve energy is an unviable option in WSNHEAP as inactivity also comes with the consequence of depleting the limited energy supply.

The concept of energy synchronization [6] has been proposed to efficiently manage the allocation and utilization of energy by sensor nodes powered by energy harvesting, taking into consideration factors like energy leakage of capacitors. The concept is realized in the TwinStar platform (Figure 8), an add-on power board that harvests energy from the environment and stores it in ultra-capacitors. The prototype has been experimentally tested in both indoor and outdoor sites, as well as, on a mobile platform (backpack).

In [14], a novel solution that dynamically synchronizes node activity patterns with available energy budget has been proposed. Specifically, they propose the Energy Synchronized Communication (ESC) which is a transparent middleware that mediates between the network layer and the data link layer to provide integrated control and timing of radio transmission activities at receiving nodes.

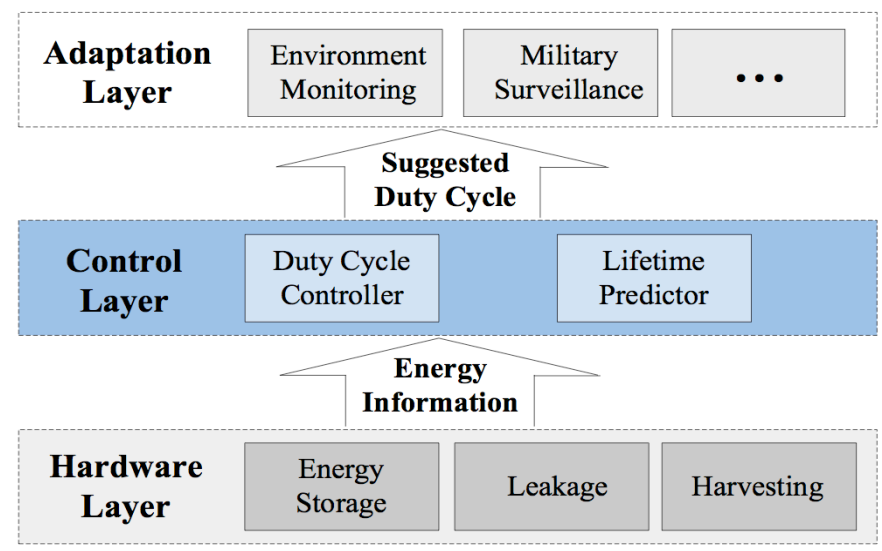

Figure 8. TwinStar System Architecture [6].

In scenarios with unknown factors and unpredictable conditions, learning methodologies are often adopted to solve complex problems. This is the approach adopted by [15] where a reinforcement learning (RL) method is applied to adapt the duty cycle of the sensor based on specified quality of service requirements, defined as heavy, moderate and light corresponding to requesting for next three timeslots operating at $100 \%$ duty cycle, next two timeslots operating at $65 \%$ duty cycle and next timeslot operating at $30 \%$ duty cycle, respectively. Simulations, assuming the use of solar energy harvesting based on the specifications of a commercial solar panel, are used to 
show that the proposed scheme is able perform better than an adaptive duty cycling scheme. Key shortcomings of this approach include the assumption that inactivity does not deplete any energy, which has been shown to be untrue [6] and the possibility of losing the learned state information in the event that memory is lost when the node runs out of energy.

Transmission power control is a key aspect of power management in WSNs. In battery-powered WSNs, tuning the transmission power is widely used to maximize the network lifetime for a given offered load. In wireless communications, varying the transmission power changes the network topology and thus has a significant impact on the performance of the networking protocols. In [16], transmit power control is used as a means to maximize throughput, reliability and fairness in a two-dimensional WSN-HEAP used in road infrastructure monitoring, as shown in Figure 9, extending their earlier work on one-dimensional WSN-HEAP used for railroad monitoring (see references in [16].)

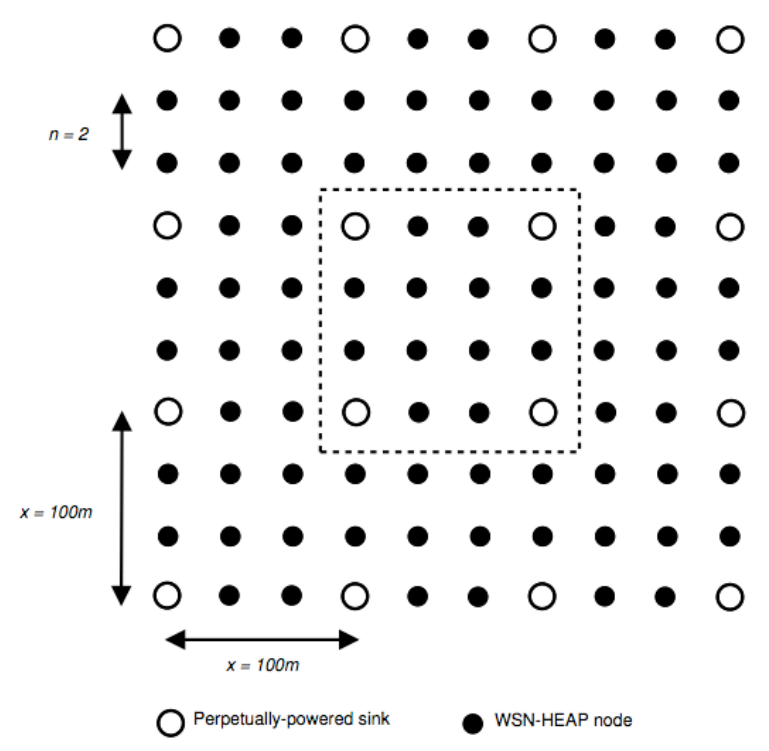

Figure 9. Two-dimensional WSN-HEAP deployment [16].

Three different power allocations schemes, namely, Fixed Transmit Power, Minimum-Interference Allocation (send only to the nearest sink) and Multi-Sink Allocation (send to $j$ of its nearest sinks, $j>2$ ), have been studied. Transmitting at higher power increases the chance of reaching a sink, but it also consumes more energy (requiring longer energy harvesting periods) and increases the level of inter-node interferences (resulting in energy wastage), both of which reduces overall throughput. In terms of throughput, the performance of the different schemes varies according to node density, while there is no clear difference among schemes in terms of reliability. The Minimum- 
Interference Allocation scheme is found to have the highest degree of fairness as its low interference level enables packets from even the furthest nodes to be successfully received by the sink.

\section{B. Data Delivery}

The process of delivering data from a sensor to the sink involves two key tasks: (i) accessing the shared wireless channel and (ii) forwarding the data to the next hop towards the sinks. Medium access control (MAC) protocols for WSNs aim to minimize interference among nodes as any transmission that is lost wastes energy. This is even more challenging in WSN-HEAP where the time taken to accumulate energy up to a useful level varies due to environmental factors as well as the energy harvesting technology used. While there are many MAC protocols designed for WSNs, they are not optimized for the unique characteristics of WSN-HEAP where nodes are unable to accurately control their wakeup schedules as the charging times are dependent on environmental conditions.

A study has been done to assess the suitability and performance of different MAC protocols when used in WSNHEAP [17]. Various Carrier Sense Multiple Access (CSMA)-based and polling-based MAC protocols have been evaluated in terms of throughput and fairness using simulations that used harvesting rate data obtained from empirical characterization of commercial energy harvesting devices. The study also investigated the impact of the maximum backoff window on an unslotted MAC scheme, as well as, derived the upper bound performance of polling schemes. Some of the key observations revealed that any scheme involving some form of backoff or retransmission is likely to be non-optimal because once a node runs out of energy timing schedules cannot be strictly enforced. Furthermore, waiting to synchronize with timeslots is counter-productive as energy is consumed during the wait period and needs to be replenished with longer harvesting periods, thus leading to lower throughputs. A single-hop probabilistic polling MAC protocol [17] is proposed after taking into consideration all these observations. In this scheme, the sink broadcasts a polling message with a polling probability value $\left(p_{\text {poll }}\right)$, and active nodes that receive this poll with generate a random number; if node's random number is smaller than $p_{\text {poll, }}$, the node will send its data, otherwise it will wait for the next poll. The sink adjusts the value of $p_{\text {poll }}$ according to network conditions, e.g. if no nodes respond, increase $p_{\text {poll, }}$, or when too many nodes respond resulting in collision/contention, decrease $p_{p o l l}$. Through analysis and simulations, it has been shown that probabilistic polling is able to achieve better performance than existing MAC protocols.

While there have been various proposed data forwarding schemes for WSNs using energy harvesting (see references in [2]), there are few schemes specifically designed to support multi-hop data delivery in WSN-HEAP 
conditions where the energy harvested is insufficient to support the desired operations. This can lead to the sensors being forced into sleep mode or worse, losing timing synchronization and data. Furthermore, since the wakeup timings of the sensor nodes cannot be predicted in advance because the charging time required is dependent on environmental factors, it is not possible for a node to know neither the number nor identity of neighbours who are able to receive packets when it is ready to transmit. Taking these factors into consideration, a multi-hop Energy Harvesting Opportunistic Routing (EHOR) protocol [18] has been proposed specifically for use in WSN-HEAP.

EHOR uses the opportunistic routing paradigm that exploits the broadcast nature of wireless transmission. The network is first partitioned into regions which are then assigned transmission priorities based on their proximity to the sink as well as the residual energy of the nodes within the regions, in order to minimize collisions while ensuring that packets advance towards the sink. The region-based approach groups nodes together to reduce delay and improve the chances of having a node that has harvested sufficient energy to receive a packet and forward it. When deciding on the transmission priority among nodes, both the residual energy in a node and its distance from the sender are used. This aims to maximize the distance covered by the packet and thus improves the probability of successfully delivering it to its destination.

Extending the probabilistic polling concept proposed in [17], Multi-Tier Probabilistic Polling (MTPP) [19] divides the WSN into multiple tiers depending on the nodes' distance from the sink. When the sink polls the first tier, nodes that are active and respond to the poll. In responding to a poll, nodes can either send data or poll nodes in the next tier that are further away or both. MTPP has been implemented on commercial-off-the-shelf devices and experimentally validated. Although the WSN prototype used in the study only involved two tiers, it is the first reported experimental study of a multi-hop WSN powered solely by energy harvesting and demonstrates the monumental challenges faced in the design of WSN-HEAP protocols that are not evident from analytical and simulation studies.

The issue of reliable data delivery in WSN-HEAP is also a critical factor considering the unpredictable energy source. The ability to collect data reliably from the network, which implies reliable data delivery, is the goal of the Probabilistic ReTransmission protocol (PRT) [20]. The approach adopted by PRT is to derive the number of times to retransmit a packet based on the reception probability and the active period of a receiver node based on its energy harvesting profile. The reception probability is computed as the proportion of time a node has been able to receive packets sent to it because it was active (after having harvested sufficient energy to operate.) A variant of PRT that 
takes into consideration the probability of packet collisions has also been proposed and both schemes evaluated using simulations.

\section{Challenges IN WSN-HEAP}

Research interest in this area is increasing exponentially and the work discussed here is by no means anywhere near comprehensive. There remain many challenges to be addressed and we briefly discuss some of them in this section.

\section{A. Correlation in natural phenomenon}

Environmental phenomena are correlated in nature and this will have a significant effect the design of the networking protocols for WSN-HEAP. For example, take the scenario where vibration and/or strain energy harvesters are used to power sensors deployed along a bridge for structural integrity monitoring. When the bridge vibrates/moves as a result of external forces, like strong winds or vehicles traveling across it, the energy harvesting process is neither random nor isolated. The energy harvesters in close proximity to one another will be excited at almost the same time, and their corresponding wireless sensors will also tend to operate in synchrony, leading to a sudden burst of network traffic. This characteristic of event-driven sensing also exists in traditional wireless sensor networks, which can be resolved through medium access control schemes and network congestion control algorithms.

However, the problem in WSN-HEAP is exacerbated by the much more severe constraint of limited energy. It has been shown in [17] that any form of backoff and retransmission will deplete the limited amount of harvested energy. Any unnecessary waiting (for slot synchronization) consumes energy and further depletes the limited supply. When there is no energy left to operate, any form of timing schedule is ineffective and data will be lost. Therefore, the desired medium access scheme should not utilize conventional approaches like backoff and retransmissions while at the same time, be able to efficiently arbitrate among the neighbouring nodes that wake up almost at the same time and operate for a short time only.

While the recently proposed schemes (e.g. [17], [18] and [20]) have considered the characteristics of WSN-HEAP and also used realistic harvesting rate data collected from experimental studies in their designs, this correlation factor has not been adequately accounted for yet.

\section{B. Modelling of Energy Harvesting Process}

It is not unexpected that majority of the recent research efforts to characterize the energy harvesting model have 
focused on solar energy. Solar energy harvesting technology has been in use for decades and the hardware can be reduced in size for use with WSNs while still providing acceptable performance. However, the use of solar energy harvesting is still subjected to the availability of light (either natural sunlight or artificial light) that is dependent on highly unpredictable environmental conditions. The high degree of variability has been observed in different characterization efforts (e.g. [22] and [23]) and shown that a general model (like the Poisson process for telephony call arrivals) is still not available, if at all. As the energy harvesting rate is very scenario, environment and technology dependent, the introduction of a scenario parameter [23] can be considered to be a first step in the right direction towards developing a viable energy harvesting model. The accuracy of the Markovian models based on the scenario parameter needs to be further validated with empirical data. In addition, the technology used for energy harvesting also plays a critical role. Comparatively less research has been done and reported on other sources of energy harvesting for explicit use in WSNs, like vibration, strain, wind, thermal, etc. This and many other factors need to be accounted for in the energy harvesting model, which will drive the design of network protocols for WSNHEAP. This area of research has just started and many problems remain to be solved especially with new energy harvesting technologies and application scenarios.

\section{Bio-inspired and Learning Approaches}

Bio-inspired methods have been widely adopted to solve complex problems in computing, communications and networking systems. When faced with situations of unpredictable conditions or no a priori knowledge, the application of bio-inspired methods becomes an attractive option.

In [24], the observation that ants rest for quite a large proportion of their time inspired their study to treat the charging cycles of WSN-HEAP (cf: Figure 2) like the resting and activity periods of ants. Duty cycling has been widely used in WSNs as a synchronization method to save energy. However, due to the unpredictable nature of energy harvesting, it is difficult to adjust the duty cycle without any a priori knowledge of the energy profile. The ability of ants to synchronize among themselves inspired this study on the feasibility of realizing self-synchronized duty-cycling for WSNs using the self-synchronization mechanisms of ant colonies. However, the study has been carried out entirely from the swarm intelligence perspective and much work is needed to adapt the scheme for use in WSN-HEAP.

Reinforcement learning (RL) is another nature-inspired method that has been widely used in computer science and engineering. It mimics the way animals adapt their behaviour from the rewards gained by performing certain 
desirable tasks and avoiding punishment from doing the wrong things. As already mentioned above, RL has been applied to provide adaptive duty-cycles for power management in WSN-HEAP scenarios [15]. However, learning requires memory of the acquired knowledge to be maintained. A key question arises on how effective are RL methods if the memory is lost when energy is depleted before it can be replenished. To mitigate this problem, some state information can be replicated on WSN-HEAP nodes in close proximity to one another so that if a node loses its memory, it can recover some of the learned knowledge from one of its neighbours.

\section{Middleware}

Middleware for WSNs aims to bridge the gap between the need for pervasive computing applications to acquire high-level sensor data and the complexity of interface access to the myriads of sensors operating within the WSNs. Typically, in providing a consistent and agreed level of quality of service, the middleware is required to dynamically adapt to changing contextual situation in resources availability and network topology. Although, several middleware research works have been reported on leveraging contextually sensed data to provide computation adaptation to pervasive applications [25][26], there is a noticeable lack of interests in applying middleware concepts to provide a service-oriented abstraction to reliably and flexibly access sensed data. The added complexity and dynamicity of WSN-HEAP over traditional WSN have accelerated the need to apply middleware services to mitigate the energy instability and low duty cycle operation of sensor nodes. In traditional WSN, energy management service of the middleware primarily focuses on minimizing energy consumption, while exercising lock-step synchronization among neighboring nodes to enforce synchronized sleep-wakeup time. However, in WSN-HEAP, it may not always be beneficial to minimize energy consumption when the energy harvested may vary significantly under different environments [14], while the energy storage devices are limited in capacity and subjected to leakage. Furthermore, the basic assumption of synchronized operation of neighboring nodes is invalidated since these nodes may not have harvested enough energy for continuous operation.

\section{CONCLUSION}

Wireless sensor networks form a significant portion of increasing number of smart devices that make up the new Internet and providing the energy to power them remains a critical problem. Energy harvesting has shown to be a plausible solution to enable sustainable sensor networks. However, the use of energy harvesting for power has introduced a new set of challenges in networking protocol design which need to address issues that are significantly 
different from those considered in the design of traditional wireless sensor networks. The key goal in the design of wireless sensor networks powered by ambient harvesting is to maximize the utility of the currently available harvested energy before it is exhausted, instead of trying to maximize the availability of a limited portable energy resource (typically a battery) and thus the operational lifetime of a sensor node.

In this paper, we first discussed recent advances in energy harvesting technology that will strongly influence the design of wireless sensor networks that rely on them for power, and the efforts to model the characteristics of some of these energy harvesting sources. This is followed by a review of research on networking protocol design for WSN-HEAP, before concluding with a discussion of open issues and challenges that remain to be addressed. We hope that this will stimulate more novel research and innovations in this exciting new area of research.

\section{REFERENCES}

[1] C.-Y. Chong and S. P. Kumar, "Sensor Networks: Evolution, Opportunities and Challenges", Proceedings of the IEEE, vol. 91, no. 8, pp. 1247-1256, August 2003.

[2] W. K. G. Seah, Z. A. Eu and H. P. Tan, "Wireless Sensor Networks Powered by Ambient Energy Harvesting (WSN-HEAP) - Survey and Challenges", Invited Paper, Proceedings of the First International Conference on Wireless Communications, Vehicular Technology, Information Technology and Aerospace \& Electronic Systems Technology (Wireless VITAE), Aalborg, Denmark, 17-20 May 2009.

[3] Y.K. Tan and S.K. Panda, "Energy Harvesting from Hybrid Indoor Ambient Light and Thermal Energy Sources for Enhanced Performance of Wireless Sensor Nodes", IEEE Transactions on Industrial Electronics, in-press, 2011.

[4] Y.K. Tan and S.K. Panda, "Optimized Wind Energy Harvesting System Using Resistance Emulator and Active Rectifier for Wireless Sensor Nodes", IEEE Transactions on Power Electronics, vol.26, issue.1, pp.38-50, 2011.

[5] R. Vullers, R. Schaijk, H. Visser, J.Penders and C. Hoof, "Energy Harvesting for Autonomous Wireless Sensor Networks", IEEE SolidState Circuits Magazine, vol. 2, no. 2, pp. 29-38, Spring 2010.

[6] T. Zhu, Z. Zhong, Y. Gu, T.He and Z.-L. Zhang, "Leakage-aware energy synchronization for wireless sensor networks", Proceedings of the $7^{\text {th }}$ International Conference on Mobile Systems, Applications and Services (MobiSys), Krakow, Poland, 22-25 June 2009.

[7] C. Alippi, R. Camplani, C. Galperti and M. Roveri, "Effective design of WSNs: From the lab to the real world", Proceedings of the 3rd International Conference on Sensing Technology (ICST)\}, Tainan, Taiwan, 30 November-3 December 2008.

[8] V. Raghunathan, S. Ganeriwal, M. Srivastava, "Emerging techniques for long lived wireless sensor networks", IEEE Communications Magazine, vol.44, issue.4, pp.108-114, 2006.

[9] D. Niyato, E. Hossain, M.M. Rashid, V.K. Bhargava, "Wireless sensor networks with energy harvesting technologies: a game-theoretic approach to optimal energy management”, IEEE Wireless Communications, vol.14, issue.4, pp.90-96, 2007.

[10] J. P. Thomas, M. A. Qidwai, and J. C. Kellogg, "Energy scavenging for small-scale unmanned systems", Journal of Power Sources, vol.159, pp.1494-1509, 2006 
[11] J.A. Paradiso, T. Starner, "Energy scavenging for mobile and wireless electronics", IEEE Pervasive Computing, vol.4, issue 1, pp.18-27, 2005.

[12] L. Laursen, "Snails in a Race for Biological Energy Harvesting", IEEE Spectrum, April 2012.

[13] S. Karavas and T.M. Fischer, "Voltree Power Leading the Way with Bio-Energy Harvesting Technology", Vaisala News, Issue 182, January 2010. Available online: http://www.vaisala.com/en/press/vaisalanews/vaisalanews182/Pages/default.aspx.

[14] Y. Gu, T. Zhu and T. He, "ESC: Energy Synchronized Communication in Sustainable Sensor Networks", Proceedings of the $17^{\text {th }}$ IEEE International Conference on Network Protocols (ICNP), Princeton, NJ, USA, 13-16 October 2009.

[15] R. Hsu, C.-T. Liu, K.-C. Wang and W.-M. Lee, "QoS-Aware Power Management for Energy Harvesting Wireless Sensor Network Utilizing Reinforcement Learning", Proceedings of the International Conference on Computational Science and Engineering (CSE), Vancouver, BC, Canada, 29-31 August 2009.

[16] H.-P. Tan, A. C. Valera and W. Koh, "Transmission Power Control in 2D Wireless Sensor Networks Powered by Ambient Energy Harvesting", Proceedings of the IEEE $21^{\text {st }}$ International Symposium on Personal Indoor and Mobile Radio Communications (PIMRC), Istanbul, Turkey, 26-30 September 2010.

[17] Z. A. Eu, H.-P. Tan and W. K. G. Seah, "Design and Performance Analysis of MAC Schemes for Wireless Sensor Networks Powered by Ambient Energy Harvesting", Ad Hoc Networks, vol. 9, no. 3, May 2011.

[18] Z. A. Eu, H.-P. Tan and W. K. G. Seah, "Opportunistic routing in wireless sensor networks powered by ambient energy harvesting", Computer Networks, vol. 54, no. 17, December 2010.

[19] C. Fujii and W. K. G. Seah, "Multi-Tier Probabilistic Polling for Wireless Sensor Networks Powered by Energy Harvesting", Proceedings of the 7th International Conference on Intelligent Sensors, Sensor Networks and Information Processing (ISSNIP 2011), Adelaide, Australia, Dec 6-9, 2011.

[20] M. Yoshida, T. Kitani, M. Bandai, T. Watanabe, P. Cai and W. K. G. Seah, "Probabilistic Data Collection Protocols for Energy Harvesting Sensor Networks", Proceedings of the 36th Annual IEEE Conference on Local Computer Networks (LCN), Bonn, Germany, Oct 4-7, 2011.

[21] Z. A. Eu, H.-P. Tan and W. K. G. Seah, "Wireless Sensor Networks Powered by Ambient Energy Harvesting: An Empirical Characterization", Proceedings of the IEEE International Conference on Communications (ICC), Cape Town, South Africa, 23-27 May 2010.

[22] P. W. Q. Lee, Z. A. Eu, M. D. Han and H.-P. Tan, "Empirical modeling of a solar-powered energy harvesting wireless sensor node for timeslotted operation", Proceedings of the IEEE Wireless Communications and Networking Conference (WCNC), Cancun, Quintana Roo, Mexico, 28-31 March 2011.

[23] C. K. Ho, P. D. Khoa and C. M. Pang, "Markovian models for harvested energy in wireless communications", IEEE International Conference on Communication Systems (ICCS), Singapore, 17-19 Nov. 2010.

[24] H. Hernandez, C. Blum, M. Middendorf, K. Ramsch and A. Scheidler, "Self-synchronized duty-cycling for mobile sensor networks with energy harvesting capabilities: A swarm intelligence study", Proceedings of the IEEE Swarm Intelligence Symposium (SIS), Nashville, TN, USA, 30 March-1 April 2009.

[25] S.-N. Chuang and A. T. S. Chan, "Dynamic QoS Adaptation for Mobile Middleware", IEEE Transactions on Software Engineering, vol. 34, no. 6, pp. 738-752, November/December 2008. 
[26] Y. Zheng, J. Cao, A. T. S. Chan and K. C. C. Chan, "Sensors and Wireless Sensor Networks for Pervasive Computing Applications", Invited Paper, Journal of Ubiquitous Computing and Intelligence, vol. 1, no. 1, pp. 17-34, 